\title{
Mixed-Signal Parallel Compressive Spectrum Sensing for Cognitive Radios
}

\author{
Zhuizhuan Yu, ${ }^{1}$ Xi Chen, ${ }^{1}$ Sebastian Hoyos, ${ }^{1}$ Brian M. Sadler, ${ }^{2}$ Jingxuan Gong, ${ }^{1}$ \\ and Chengliang Qian ${ }^{1}$ \\ ${ }^{1}$ Department of Electrical and Computer Engineering, The Analog and Mixed Signal Center (AMSC), Texas A\&M University,
College Station, TX 77843-3128, USA
${ }^{2}$ Army Research Laboratory, AMSRD-ARL-CI-CN, Adelphi, MD 20783, USA
}

Correspondence should be addressed to Sebastian Hoyos, hoyos@ece.tamu.edu

Received 2 December 2009; Accepted 30 January 2010

Academic Editor: Fred Daneshgaran

Copyright (C) 2010 Zhuizhuan Yu et al. This is an open access article distributed under the Creative Commons Attribution License, which permits unrestricted use, distribution, and reproduction in any medium, provided the original work is properly cited.

\begin{abstract}
Wideband spectrum sensing for cognitive radios requires very demanding analog-to-digital conversion (ADC) speed and dynamic range. In this paper, a mixed-signal parallel compressive sensing architecture is developed to realize wideband spectrum sensing for cognitive radios at sub-Nqyuist rates by exploiting the sparsity in current frequency usage. Overlapping windowed integrators are used for analog basis expansion, that provides flexible filter nulls for clock leakage spur rejection. A low-speed experimental system, built with off-the-shelf components, is presented. The impact of circuit nonidealities is considered in detail, providing insight for a future integrated circuit implementation.
\end{abstract}

\section{Introduction}

Cognitive Radio (CR), first proposed in [1], provides a new paradigm to improve spectrum efficiency by enabling Dynamic Spectrum Access (DSA). In CR, spectrum holes that are unoccupied by primary users can be assigned to appropriate secondary users as long as the interference introduced by secondary users is not harmful to the primary users [2-4]. The design of cognitive radio networks is a complicated cross-layer procedure [5]. In this paper, we focus on the spectrum sensing problem in $\mathrm{CR}$, in which sensing and detection of primary users is done in order to realize Dynamic Spectrum Access.

Spectrum sensing can be a very challenging task for CR due to many factors. First, for the sake of improving the frequency usage efficiency, the sensing bandwidth for CR can expand from hundreds of $\mathrm{MHz}$ to several GHz. Second, the sensing radio should be able to detect very weak primary users, which arise due to fading and the hidden terminal problem [5]. With traditional time-domain Nyquist sampling, sensors are needed with both wide bandwidth and high dynamic range, stressing technology, and demanding higher power $[6,7]$. Conventional wideband sensing with a high- speed and high-resolution ADC becomes less appealing as the bandwidth becomes significant. Alternative approaches, such as a fixed bank of analog filters followed by parallel ADCs, impose strict requirements on the filter design.

It has been observed that today's spectrum usage presents some sparsity in the sense that only a small portion of the available frequency bands are heavily loaded while others are partially or rarely occupied [5]. This frequency usage sparsity can be exploited under the framework of Compressed Sensing (CS) $[8,9]$ to effectively reduce the sampling rate. The sparse signal can be captured via projection over a random basis that is incoherent with respect to the signal basis, and perfect signal reconstruction from these projections can be obtained with high probability, where the number of random projections is on the order of the signal's information rate rather than the Nyquist rate.

The idea of applying CS for wideband spectrum sensing was reported, for example, in [10]. However, this approach assumes full-rate analog-to-digital conversion which does not reduce the complexity of the spectrum sensing receiver. We have proposed a mixed-signal parallel segmented compressive sensing (PSCS) architecture for wideband spectrum sensing [11], where the high-speed ADCs were avoided by 
carrying out an analog basis expansion in parallel before sampling. In this paper we elaborate on the idea of applying the PSCS front-end [11], with special emphasis on implementation issues such as spurious frequency tones, timing, and other mismatches. First, we show that the proposed overlapping windowed integration in the PSCS architecture provides a scheme to mitigate the spurs due to clock leakage by setting the lowpass filter nulls flexibly, which is favorable for practical implementation. Second, a low-speed prototype built with off-the-shelf components is presented in detail from the overall system configuration to building blocks, in which practical constraining issues are addressed.

The remainder of the paper is organized as follows. A brief background on CS is provided in Section 2 and the spectral occupancy signal modeling is given in Section 3. Section 4 introduces the mixed-signal parallel compressive spectrum sensing scheme. Section 5 discusses the spurious frequency rejection schemes in the PSCS front-end. A lowspeed prototype is introduced in Section 6. Conclusions are made in Section 7.

\section{Compressive Sensing Background}

A signal $r(t)$ that is spanned by $S$ basis functions $\Psi_{s}(t)(s=$ $1,2, \ldots, S)$, that is, $r(t)=\sum_{s=1}^{S} a_{s} \Psi_{s}(t)$, or in the matrix form $\mathbf{r}=\Psi \mathbf{a}$, is a $K$-sparse signal if only $K$ out of the $S$ coefficients $\left.a_{s}\right|_{s=1} ^{S}$ are nonzero at any time, where $K \ll S$. A signal $r(t)$ is compressible if its approximation error by a $K$-sparse signal decays exponentially as $K$ increases.

According to CS theory, a signal that is sparse or compressible over a known basis $\Psi$ can be sampled and reconstructed at sub-Nyquist rate, and the sampling rate reduction depends on the signal's sparsity and the reconstruction algorithms. Specifically, the sub-Nyquist rate sampling is achieved by projecting the signal into a transform-domain over which the sampling operation occurs, which is different from the traditional way of sampling the signal in the timedomain. Mathematically, this procedure can be described as $\mathrm{y}=\boldsymbol{\Phi} \boldsymbol{\Psi} \mathbf{a}$, where $\mathrm{y}$ are the collected samples, and $\boldsymbol{\Phi}$ is incoherent with $\Psi$ which is the basis for the transformdomain. The reconstruction of the original signal relies on the estimation of the coefficients a, which is obtained by solving the following $l_{1}-$ norm optimization problems, for which many convex optimization techniques or iterative greedy algorithms can be used:

(i) noiseless case:

$$
\widehat{\mathbf{a}}=\arg \min \|\mathbf{a}\|_{1} \quad \text { s.t. } \mathbf{y}=\boldsymbol{\Phi} \Psi \mathbf{a},
$$

(ii) noisy case:

$$
\hat{\mathbf{a}}=\arg \min \|\mathbf{a}\|_{1} \quad \text { s.t. }\|\mathbf{y}-\boldsymbol{\Phi} \Psi \mathbf{a}\|_{2} \leq \epsilon,
$$

where $\epsilon$ is the error due to the noise.

Note that, in this paper, we generally do not differentiate between sparse and compressible unless specifically noted.

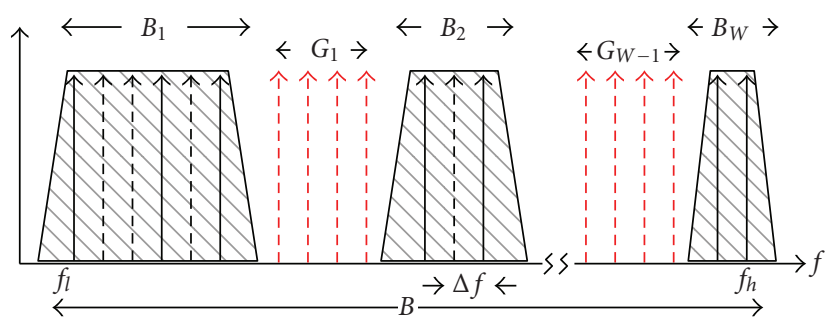

FIGURE 1: Illustration of the multiband analog signal to the sensing radio.

\section{Signal Modeling}

The received signal $r(t)$ is a modeled as a multiband analog signal whose spectrum is illustrated in Figure 1. Specifically, we assume that $r(t)$, with a frequency span from $f_{l}$ to $f_{h}$, is the superposition of primary users, perhaps using $W$ different wireless standards [5]. Each wireless standard occupies a certain finite frequency band which consists of multiple channels. According to the measurements done by FCC in the US [12], in many cases the current frequency usage exhibits sparsity because only a part of the allocated channels is utilized at a given time.

Without loss of generality, we assume that $r(t)$ is bandlimited to $\left[0, f_{h}\right]$; so $r(t)$ can be written as

$$
r(t)=\int_{-\infty}^{\infty} R(f) e^{j 2 \pi f t} d f=\int_{0}^{f_{h}} R(f) e^{j 2 \pi f t} d f
$$

where $R(f)$ is the Fourier transform of $r(t)$.

The continuous-time analog signal can be captured with a finite dimensional model; for example, see $[13,14]$. We directly approximate $r(t)$ with a model of finite dimension as follows:

$$
r(t) \approx \sum_{s=0}^{S-1} R(s \Delta f) e^{j 2 \pi s \Delta f t} \Delta f, \quad t \in\left[0, T_{s}\right]
$$

where $\Delta f=1 / T_{s}$ is the resolution on the frequency axis and $(S-1) \Delta f=f_{h}$. In other words, $r(t)$ is approximated as a multicarrier signal bandlimited to $\left[0, f_{h}\right]$ and with a carrier spacing of $\Delta f$. The sparse frequency occupancy means that statistically, speaking, only $K$ out of the $S$ carriers are active at any time, where $K \ll S$. The multicarrier model is convenient for representing user occupancy with spectral sparsity. Comparing (3) and (4), we notice that this model is based on a finite dimensional approximation of the signal spectrum. Since there are $S$ unknowns where $R(s \Delta f)$ in (4) and $R(s \Delta f)$ change every $T_{s}$ seconds, the model in (4) is a case of a Finite Rate of Innovation (FRI) model in which the innovation locations lie on the Nyquist grid. For clarity, we rewrite (4) as

$$
r(t)=\sum_{s=0}^{S-1} a_{s} \Psi_{s}(t)+n(t)
$$




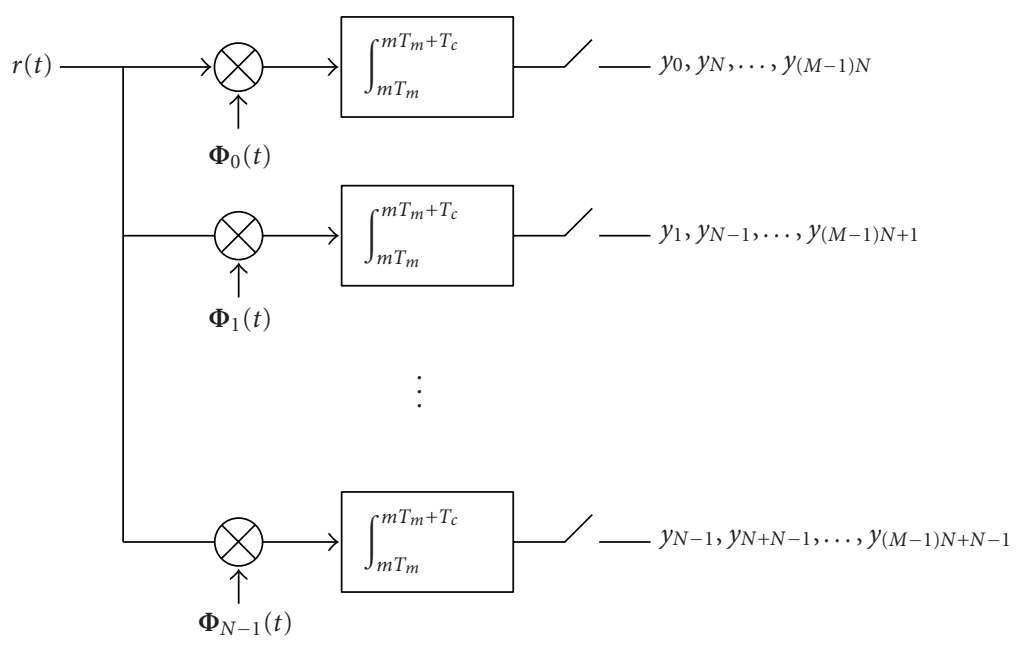

FigURE 2: Block diagram of the parallel segmented compressive sensing (PSCS) architecture.

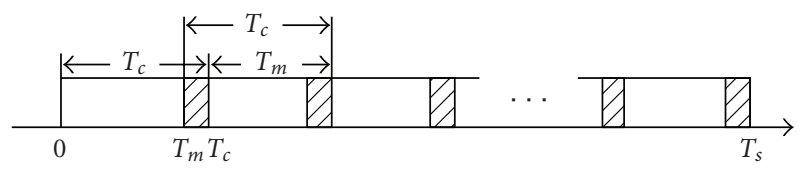

FIGURE 3: Illustration of overlapping windows.

where $n(t)$ is additive white Gaussian noise (AWGN), $\Psi=\left[\Psi_{0}(t), \Psi_{1}(t), \ldots, \Psi_{S-1}(t)\right], \Psi_{s}(t)=e^{j 2 \pi s \Delta f t}, \mathbf{a}=$ $\left[a_{0}(t), a_{1}(t), \ldots, a_{S-1}(t)\right] \in \mathbb{C}^{S}, a_{s}=\Delta f R(s \Delta f)$, and a has only $K \ll S$ nonzero elements. Since $\Delta f$ is a scalar, for simplicity, we discard it in the rest of the paper. The spectrum hole detection, for example, energy or feature detection, is usually based on the observed signal spectrum $R(s \Delta f)$, or equivalently, the estimation of the coefficients $a_{s}$.

\section{Wideband Parallel Compressive Spectrum Sensing}

Wideband spectrum sensing is composed of several crucial steps: first, spectrum estimation; second, calculate the sufficient statistics, during which digital signal processing is needed to improve the front-end sensing sensitivity by processing gain and identification of the primary users based on knowledge of the signal characteristics [5]; last, to decide whether there exist primary users based on the sufficient statistics. Here we focus on the wideband spectrum estimation step, that is, estimating the unknown coefficients $\mathbf{a}$ in (5).

4.1. Mixed-Signal Compressive Sensing Architecture. The parallel segmented compressive sensing (PSCS) structure is shown in Figure 2, which we first proposed in [11]. For the completeness of this paper, in this section we recap how the analog compressive sensing at sub-Nyquist rate is realized via the PSCS architecture.

In the PSCS architecture, the input signal $r(t)$ is sent to $N$ parallel paths. In the $n_{\text {th }}$ path, $r(t)$ is mixed with a random basis function $\Phi_{n}(t)$. A good choice for the random basis is to use PN (Pseudonoise) sequences because they can be conveniently generated by digital logic circuits. The output of the mixer is then sent to a sliding window with a width of $T_{c}$ and integrated. Two adjacent windows have an overlapping time $T_{c}-T_{m}$, which defines an overlapping percentage OVR $=\left(T_{c}-T_{m}\right) / T_{c}$, as shown in Figure 3. The output of the integrators is sampled and $M$ samples are collected at each path. The $m_{\text {th }}$ sample of the $n_{\text {th }}$ branch is given by

$$
y_{m N+n}=\int_{m T_{m}}^{m T_{m}+T_{c}} r(t) \Phi_{n}^{*}(t) d t .
$$

There are a total of $L=M N$ samples collected every $T_{s}$ seconds and these samples are organized into a vector as follows:

$$
\mathbf{y}=\left[\tilde{\mathbf{y}}_{0}^{T}, \tilde{\mathbf{y}}_{1}^{T}, \ldots, \tilde{\mathbf{y}}_{M-1}^{T}\right]^{T}
$$

where $\tilde{\mathbf{y}}_{\mathbf{m}}=\left[y_{m N}, y_{m N+1}, \ldots, y_{m N+N-1}\right]^{T}$ is the vector consisting of the $m_{\text {th }}$ samples from all $N$ branches.

Similarly, we can calculate the reconstruction matrix $\mathbf{V}=$ $\Phi \Psi=\left\{v_{i, j}\right\}_{L \times S}$. The element at the $m N+n$ row and the $s$ column is given by

$$
V_{m N+n, s}=\int_{m T_{m}}^{m T_{m}+T_{c}} e^{j 2 \pi s \Delta f t} \Phi_{n}^{*}(t) d t .
$$

Therefore, we have $\mathbf{y}=$ Va. Then, we can estimate a by solving the problem in (1) and reconstruct the original signal using $\mathbf{r}=\Psi \hat{\mathbf{a}}$.

4.2. A Wideband Spectrum Sensing Example. To show the effectiveness of the proposed wideband PSCS architecture we present a simulation, where the input signal is modeled as a frequency-domain sparse multi-carrier signal as given in (5). The mixed-signal compressive sensing based on the PSCS architecture given in Figure 2 is used for spectrum 


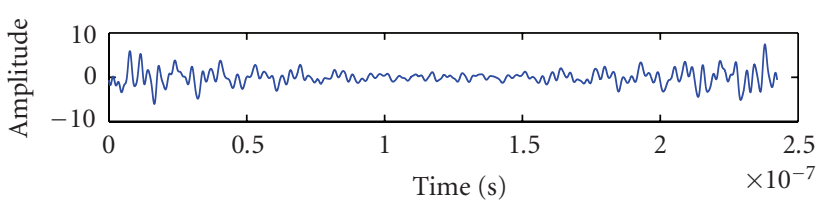

- Transmitted

(a)

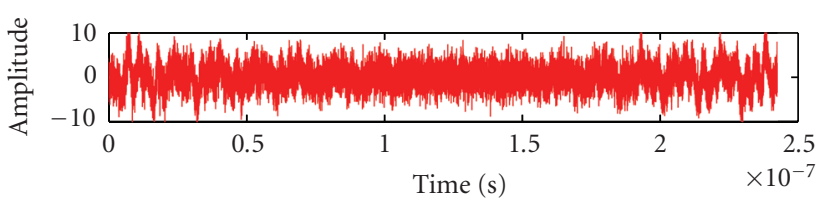

Received

(b)

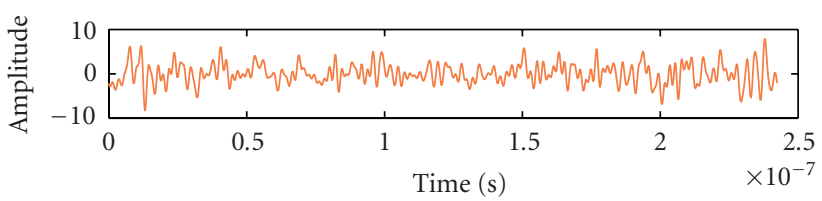

— Reconstructed via Nyquist FFT

(c)

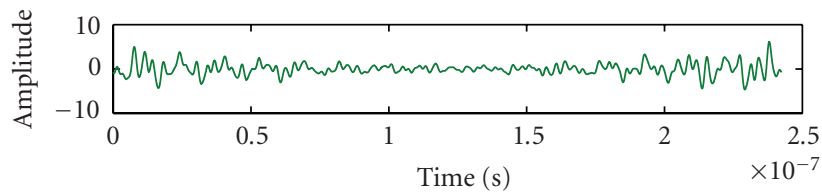

_ Reconstructed via sub-Nqyuist CS

(d)

Figure 4: Time-domain signals of a simulated multiband signal. From top to bottom, the four plots represent the transmitted signal by primary users, the received primary users' signal at the sensing radio, the reconstructed signal from the time-domain samples via the Nyquist rate $\mathrm{ADC}$, and the reconstructed signal from the transform-domain samples via mixed-CS at an NSR of 0.32 .

estimation. The sampling rate reduction is measured by the Normalized Sampling Rate (NSR), which is defined as

$$
\mathrm{NSR}=\frac{f_{C S}}{f_{N q}}=\frac{M N}{S},
$$

where $f_{C S}$ is the sampling rate required using the PSCS and $f_{N q}$ is the corresponding Nyquist sampling rate. The signal reconstruction quality is evaluated by the normalized Mean Square Error (MSE), which is equal to

$$
\frac{\|\mathbf{a}-\hat{\mathbf{a}}\|_{2}}{\|\mathbf{a}\|_{2}}
$$

In the simulation, the input signal to the PSCS architecture is assumed to be a 17-sparse frequencydomain multi-carrier signal with 128 subcarriers, that is,

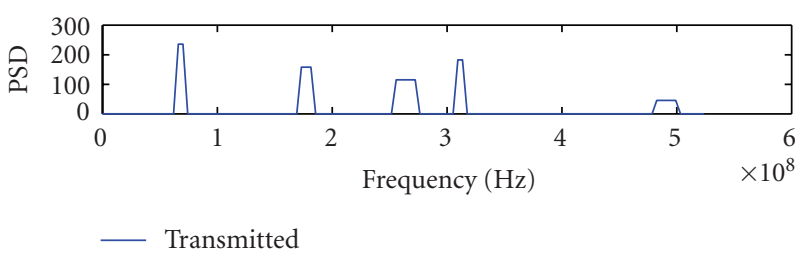

(a)

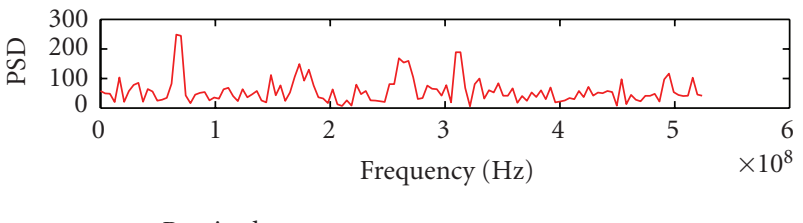

(b)

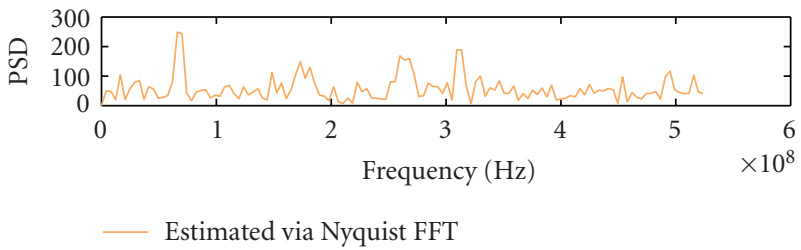

(c)

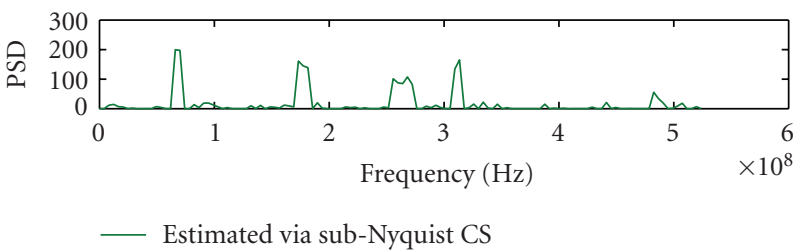

(d)

FIGURE 5: Frequency-domain signals of a simulated multiband signal. From top to bottom, the four plots represent the transmitted signal by primary users, the received primary users' signal at the sensing radio, the reconstructed signal from the time-domain samples via the Nyquist rate $\mathrm{ADC}$, and the reconstructed signal from the transform-domain samples via mixed-CS at a NSR of 0.32 .

$S=128$ and $K=17$. There are 5 primary bands with an overall bandwidth of $528 \mathrm{MHz}$. The subcarrier spacing $\Delta f=528 \mathrm{MHz} / 128=4.125 \mathrm{MHz}$ and the primary user's frequencies are $(17,18,43,44,45,63,64,65,66,67$, $76,77,118,119,120,121,122) \times 4.125 \mathrm{MHz}$. The input power dynamic range of the primary users is $15 \mathrm{~dB}$. $\mathrm{SNR}_{\text {overall }}=0 \mathrm{~dB}$, where $\mathrm{SNR}_{\text {overall }}$ is the total signal power over the whole bandwidth divided by the total noise power over the whole bandwidth. (Note how noisy the received signal is in this example, shown in Figure 4.) In Figures 4 and 5, from top to bottom, the four plots represent the primary transmitted signal, the received primary users' signal at the sensing radio, the reconstructed signal from the time-domain samples via the Nyquist rate ADC, and the reconstructed signal from the transform-domain samples via mixed-CS at an NSR of 0.32. The measured MSE for the two reconstructed signals is $-5 \mathrm{~dB}$ and $-14 \mathrm{~dB}$, respectively. Note that even with a lower sampling rate, the sensing radio 


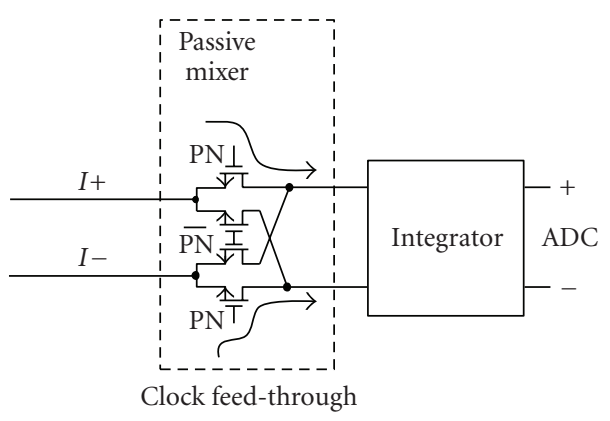

FIgURE 6: Clock leakage into the integrators from the clock of the $\mathrm{PN}$ generators.

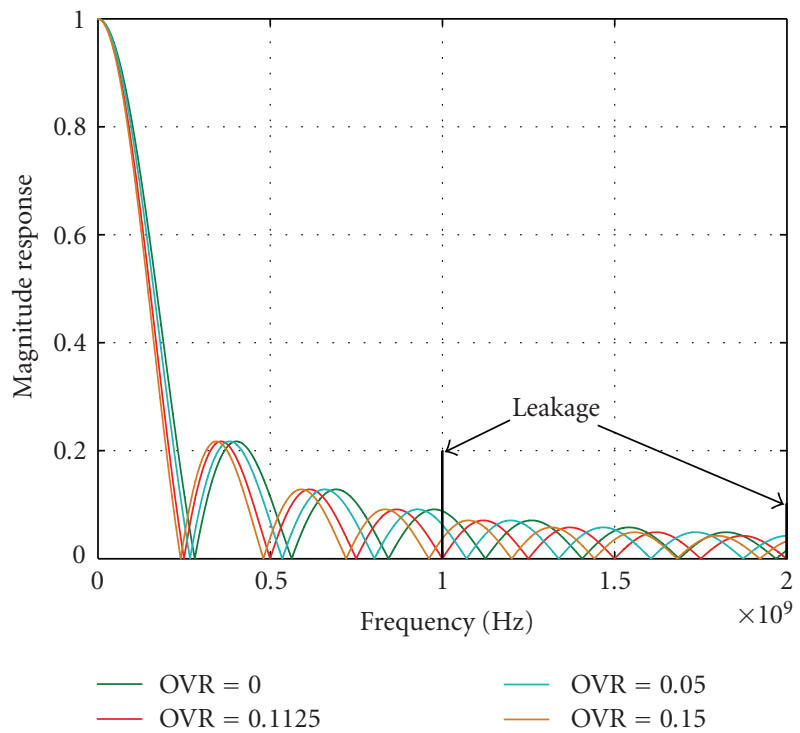

FIGURE 7: The location of the spurious leakage frequency relative to the filter nulls with different overlapping ratio. With OVR $=0$, the strongest clock leakage is close to the peak of the filter's 3rd sidelobe; with OVR $=0.1125$, the strongest clock leakage is on the 4 th null of the filter.

based on mixed-signal PSCS is more robust against noise than the traditional digital approach based on the DFT, because CS takes advantage of the knowledge of the signal structure and its sparsity.

\section{Flexible Spur Rejection via the Overlapping Windowed Integration}

In addition to the capability of sensing and reconstructing sparse signals at sub-Nqyuist rate, the PSCS architecture has many special characteristics. For example, the parallel architecture gives a design tradeoff between the sampling rate and the system complexity [11]. In this section, we focus on the PSCS architecture's spurious frequency rejection schemes. Since one critical type of spur in the PSCS architecture is the leakage of the clocks for the PN generators to the integrator, as illustrated in Figure 6, we will focus on this particular type of spur in this section, although the rejection scheme applies more generally.
Recall that in Figure 2, the output after the mixer is sent to a sliding window with a width of $T_{c}$ and integrated over $T_{c}$ seconds, and there is an overlap time of $T_{c} \times$ OVR between two adjacent windows as illustrated in Figure 3. The integrator, with a reset every $T_{c}$ seconds, provides a simple realization of a sinc type lowpass filter with nulls at frequencies of $f_{0} \times k$, where $f_{0}=1 / T_{c}$. By setting the random generator clock frequency equal to a harmonic of the reset frequency, the sinc nulls coincide with spur frequencies from the random generator clock and so filters them, where the overlapping scheme provides the flexibility on setting the locations of the nulls. In some cases, without the overlapping scheme, the objective of setting the clock frequency on the nulls of the sinc type lowpass filter may conflict with the sampling rate requirement which is determined by the signal's sparsity. In order to show this, consider the following example.

Let the input signal to the PSCS architecture be a 19-sparse frequency-domain multi-carrier signal with 128 subcarriers, that is, $S=128$ and $K=19$, which corresponds to a sparsity of $15 \%$. The subcarrier spacing is $\Delta f=$ $1 \mathrm{GHz} / 128=7.8125 \mathrm{MHz}$ and the symbol duration time is $T_{s}=1 / \Delta f=128$ nanoseconds. The locations of the $K$ active subcarriers are chosen randomly and changed every $T_{s}$ seconds. According to simulation results, the minimum NSR is $0.5625=72 / 128$ for this parameter setup. Equivalently speaking, 72 samples are needed per 128 ns to reconstruct the signal perfectly. Using two parallel paths, 36 samples are collected every 128 nanoseconds at each path, that is, $M=36$ and $N=2$. With this parameter setup and without the overlapping scheme, $T_{c}=T / M=128 / 36=3.56 \mathrm{~ns}$, $f_{0}=1 / T_{c}=281.25 \mathrm{MHz}$, and the nulls of the sinc type lowpass filter occur at $k \times 281.25 \mathrm{MHz}$.

There may exist some leakage into the integrators from the clock signal, as illustrated in Figure 6. According to the CS theory, the clock frequency is usually at the Nyquist frequency $f_{N q}$ where $f_{N q}=1 \mathrm{GHz}$ in this example. Because $f_{N q} / f_{0} \approx 3.56$, the spurs due to the clock leakages will fall near the 3rd sidelobe's peak of the sinc type lowpass filter and bring distortion to the reconstructed signal. With the overlapping scheme, we can choose $T_{c}=4 \mathrm{~ns}$ and $f_{0}=$ $250 \mathrm{MHz}$ by introducing an overlapping ratio of $11.43 \%$, then $f_{N q} / f_{0}=4$ and the spurs due to the clock leakage can be filtered (considering the clock resolution requirement, an overlapping ratio of $11.25 \%$ is suggested in practice.). Based on Figure 3, this can be mathematically expressed as

$$
\begin{aligned}
T & =T_{c}(M-(M-1) \mathrm{OVR}), \\
f_{N q} & =\frac{S}{T}, \\
f_{0} & =\frac{1}{T_{c}}, \\
\Longrightarrow \frac{f_{N q}}{f_{0}} & =\frac{S}{M-(M-1) \mathrm{OVR}} .
\end{aligned}
$$

According to (14), given a desired sampling rate, or equivalently speaking, a specific $M$, varying OVR will change the 


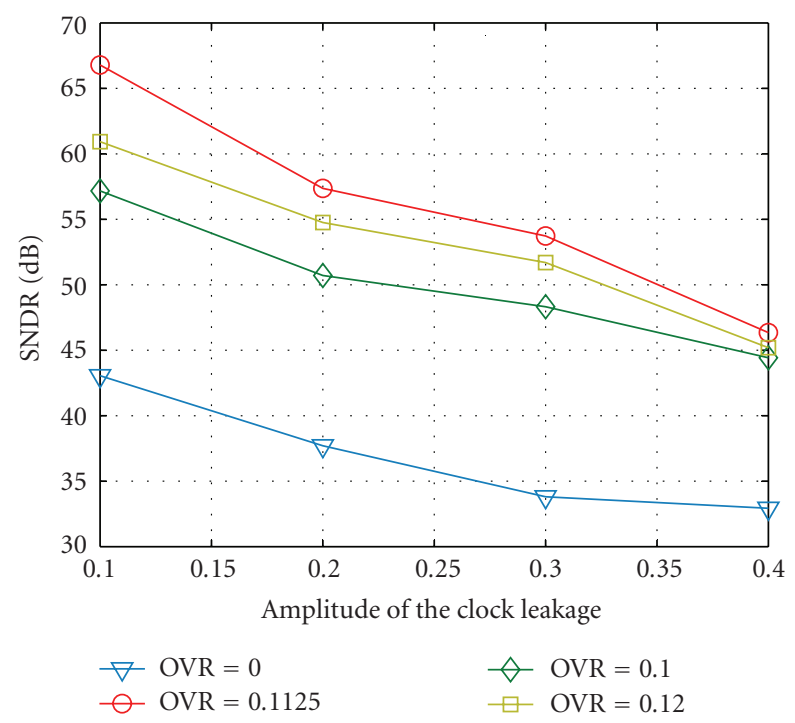

FIGURE 8: MSE of the reconstructed signal with different overlapping ratio when there is clock leakage, where the input signal is an 18-sparse multi-carrier signal with 128 possible subcarriers and sampled by the 2-path PSCS working at $56.25 \%$ of the Nyquist rate.

relative location of the leakage frequency to the filter nulls, as illustrated in Figure 7.

Note that if we do not want to introduce any overlapping but still wish to null out the clock leakage, the only option in the above example is to increase the sampling rate and make $f_{N q} / f_{0}$ an integer no less than โ3.56」. By introducing a nonzero OVR, we can conveniently make $f_{N q} / f_{0}$ an integer without increasing the sampling rate.

Figure 8 shows the MSE of the reconstructed signal versus the overlapping ratio when there is some clock leakage into the integrators. Note that in the simulation the amplitude of each subcarrier is set to 1 . Since $K=$ 19 , the signal's peak amplitude is 19 . Allowing a $10 \mathrm{~dB}$ margin to account for the multi-carrier signal's large peakto-average ratio, the clock leakage with an amplitude of 0.1 (0.4) is roughly $35 \mathrm{~dB}(23 \mathrm{~dB})$ below the signal's average power. As shown in Figure 8, the flexibility of setting the null frequencies by the overlapping scheme can bring about $20 \mathrm{~dB}$ gain after filtering the spurs due to the clock leakage.

Note also that the overlap in the integration windows provides wider filter nulls than the sinc filter. Because of the existence of the phase noise on the clock signal in practice, even if we can set the clock on the null frequency, it is inevitable that remains some leakage due to the widening of the spurs spectrum. The wider nulls provides the possibility of further improving the harmonic rejection when the phase noise is significant.

\section{Low-Speed Off-the-Shelf Component Prototype}

As a proof of concept, we built a low-speed prototype using off-the-shelf components, where the input signal is a real BPSK modulated multi-carrier signal with 4 active subcarriers and the active subcarriers hop over the frequencies

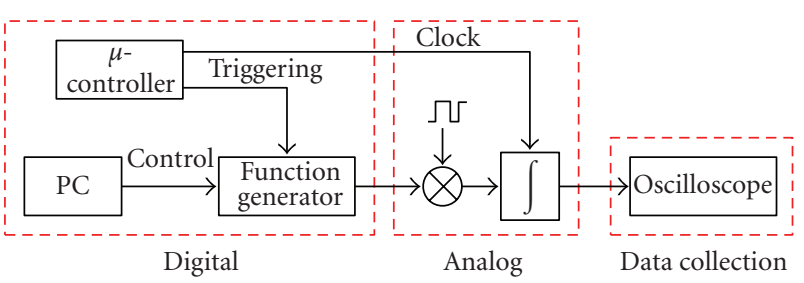

FIGURE 9: Overall configuration of the prototype using off-the-shelf components.

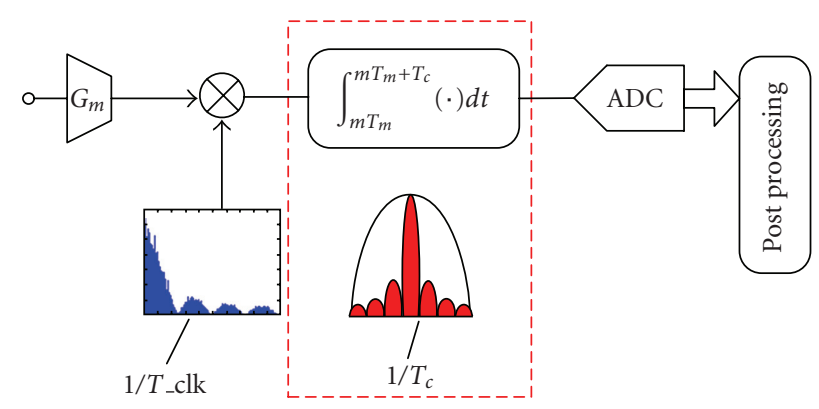

Figure 10: Macro model of one parallel path.

$(i * 2-1) \mathrm{KHz}(i=1,2, \ldots, 100)$ every 500 microseconds. Considering the system complexity, we employed 4 parallel paths for the prototype. Simulation shows that the signal can be reconstructed perfectly when each parallel path produces 16 samples every 500 microseconds, which corresponds to $32 \%$ of the Nyquist sampling rate.

6.1. Overall Configuration. The overall configuration of the prototype is shown in Figure 9, where the digital part is responsible for generating the input sparse signal, the triggering signal, the pseudorandom basis, and the clock. The analog part is used to realize the random basis projection that is essential for the signal reconstruction. The built-in ADC in the oscilloscope is used to collect the sampled data. Then, the collected data is sent to a PC and processed via Matlab code to reconstruct the signal. In the following sections, each building block will be introduced in detail.

6.2. Multicarrier Signal Generator. An Agilent 33120A arbitrary waveform generator is used to generate the input multitone sparse signal. Specifically, the multitone signal is programmed in the PC first and then downloaded into the wave generator. The output port of the generator is triggered by the microcontroller in order to synchronize with the integrator clock that is also generated by the microcontroller.

6.3. Mixers and Integrators. Figure 10 depicts the macromodel of one path in the prototype. As shown, the input signal is first translated into current by the OTA and then mixed with the pseudorandom signal. After mixing, the signal is integrated in the sampling capacitor with a timing window. In the sampling circuit the interleaving capacitor is employed. Finally the ADC yields digital output data. The OTA we employed is a TIOPA861 with Gm of $116 \mathrm{mS}$ 


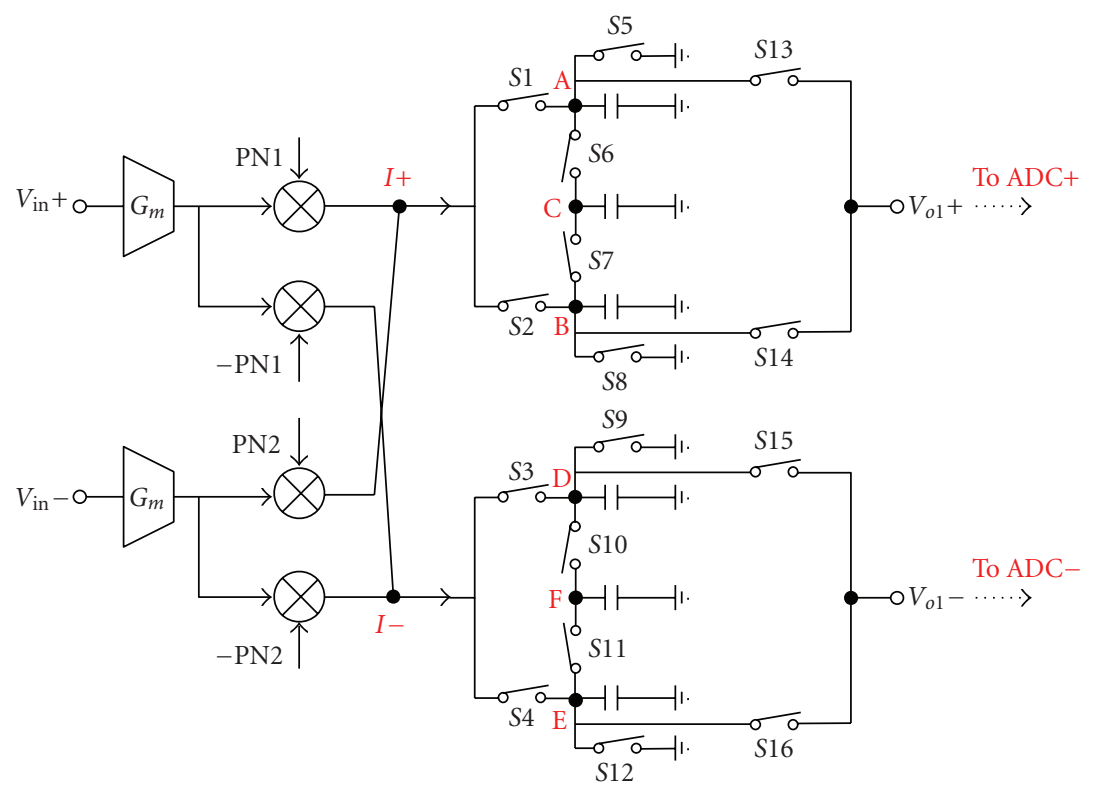

FIGURE 11: Circuit implementation of one parallel path.
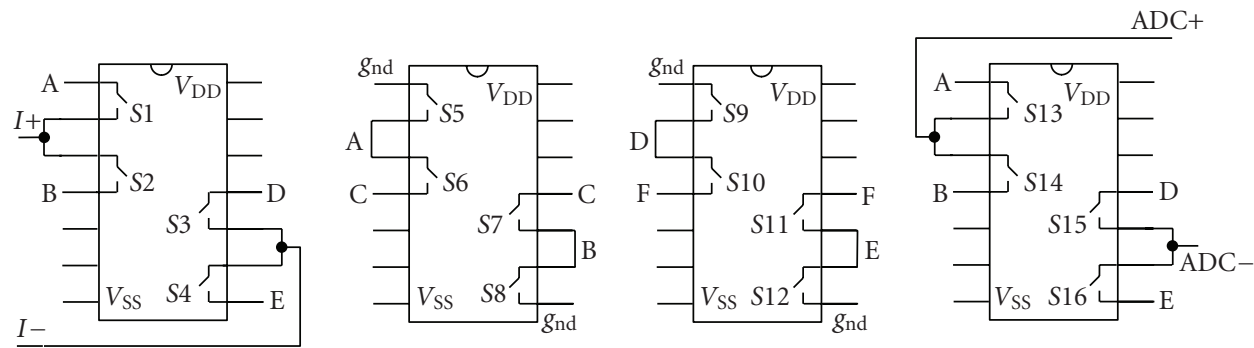

FIGURE 12: Pin connection of the integrator in one parallel path.

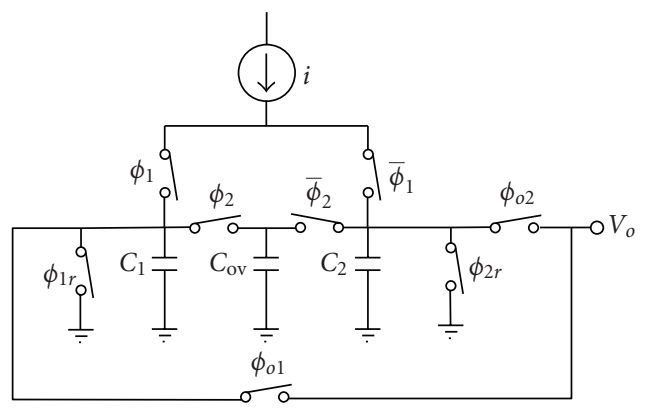

FIGURE 13: Schematic of the integrator with overlapping.

and all the switches are implemented with transmission gate CD4066BCN.

The pseudorandom number $(\mathrm{PN})$ is -1 or 1 , whose spectrum is a sinc function. The main lobe is from 0 to $1 / T_{\mathrm{clk}}$, where $T_{\text {clk }}$ is the clock period of the PN generator. In our test bed $T_{\text {clk }}$ is $1 \mu \mathrm{s}$. After the mixing, the signal is shaped by the embedded lowpass filter provided by the integration window. The frequency response of the LPF is a sinc function. The main lobe spans $1 / T_{c}$, where $T_{c}$ is the integration time. In our test bed the $1 / T_{c}$ is roughly $30 \mathrm{KHz}$.

The random projection of the input analog signals is realized with mixers and integrators. Figure 11 gives the circuit implementation of one parallel path and Figure 12 gives the corresponding pin connection relationship for the integrator. The transconductance amplifier $\left(G_{m}\right.$ stage) translates the signal voltage into current, which can be easily mixed with the pseudorandom numbers $(1 /-1)$ by the following passive switch mixer. After mixing, the signal is integrated with an overlapping window and then sampled by the ADC in each path. The circuit is built up differentially so that the system is more robust to supply noise, clock jitter, and even-order harmonics. The double balanced passive mixer does not introduce significant noise and distortions.

At each path, the mixer consists of transmission-gate switches controlled by $\mathrm{PN}$ sequences. The PN sequence is implemented with a linear feedback shift register (LFSR). In our prototype, the clock frequency is chosen to be $1 \mathrm{MHz}$, which is higher than the Nyquist sampling rate. Because the PN sequences are repeated every $500 \mu$ s and there are 4 parallel paths, we need 4 independent $\mathrm{PN}$ sequences with a length of 500. An 11-bit LFSR is used to generate a PN sequence with a length of 2047 and then divided into 4 segments. As a check, the autocorrelation function of the $\mathrm{PN}$ sequences is calculated to make sure that the four PN sequences are incoherent.

An overlapped time-interleaving charge-domain sampling integrator is chosen for the analog path. The 


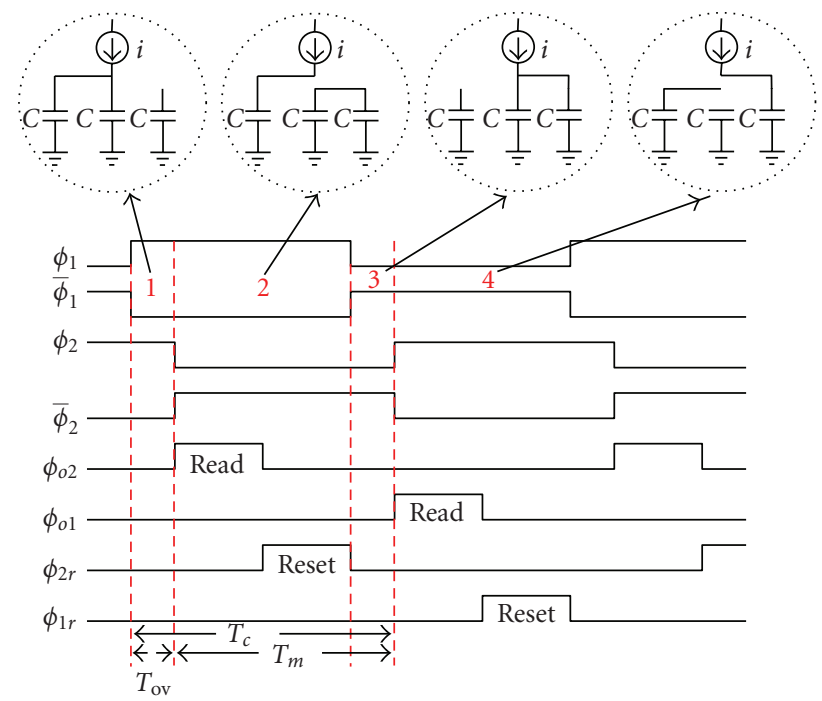

FIGURE 14: Operation of the interleaved overlapping windowed integration and the related clocks.

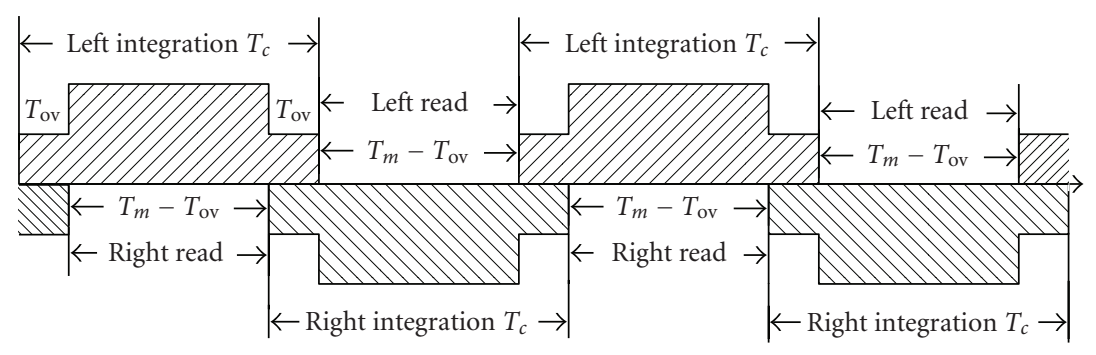

FIGURE 15: Illustration of the overlapping windowing in the prototype.

integrator schematic is shown in Figure 13, $\phi_{1}$ and $\bar{\phi}_{1}$ are two integration switches for the left and right branches, respectively. $\phi_{o 1}$ and $\phi_{o 2}$ are readout switches; $\phi_{r 1}$ and $\phi_{r 2}$ are reset switches. By utilizing these six switches combined with the two integration capacitors $C_{1}$ and $C_{2}$, according to the clock diagram shown in Figure 14, we can realize a conventional time-interleaving charge-domain integrator without overlapping. Time interleaving means when the left branch is integrating while the right is reading out, and vice versa. By doing this, a complete sampling of the signal is achieved. In addition to time interleaving, a small overlapping time is introduced by one more capacitor $C_{o v}$ and two control switches $\phi_{2}$ and $\bar{\phi}_{2}$.

As shown in Figure 14, phase1 and phase 3 are to realize the overlapping through charge redistribution and sharing, and phase 2 and phase 4 are the readout times for the right and the left branches, respectively. During phase1, the input current charges both $C_{1}$ and $C_{o v}$ while $C_{2}$ is idle. Since all capacitors have the same value, the current splits equally by half into both capacitors. In the succeeding phase, $C_{o v}$ is switch-connected to $C_{2}$ and readout together, so that $C_{o v}$ is integrating for the right branch during phase1. Equivalently, as shown in the timing window diagram, the window splits by half during the overlapping time. The key point here is that both branches are integrating and no data is readout during window overlapping times.
Note that the overlapping windowing realized using the circuit in Figure 13 is somewhat different from the overlapping windowing in Figure 3, as shown in Figure 15. In Figure 3, the charges accumulated during the current window period include $100 \%$ of the charges from the last $T_{o v}$ seconds of the previous windowing period but no charges from the next windowing period. In Figure 13, the charges accumulated during the current windowing period include $50 \%$ of the charges from both the last $T_{o v}$ seconds of the previous windowing period and the first $T_{o v}$ seconds of the next windowing period, which is more realistic from the implementation perspective.

6.4. Data Collection and Signal Reconstruction. For simplicity, we use the inherent ADC of the oscilloscope (Tectronix TDS $3054500 \mathrm{MHz}, 5 \mathrm{Gs} / \mathrm{s}$ ) to sample the output of the integrators. The sampled data is transferred to the PC via the GBIP port. With the collected samples, the signal is reconstructed as described in Section 2.

6.5. Dealing with Circuit Nonidealities. While implementing the prototype, it is inevitable that the system has some nonidealities such as the delay caused by each component, the gain variation, and the mismatch among parallel paths. Considering all the nonideal factors, the actual relationship 

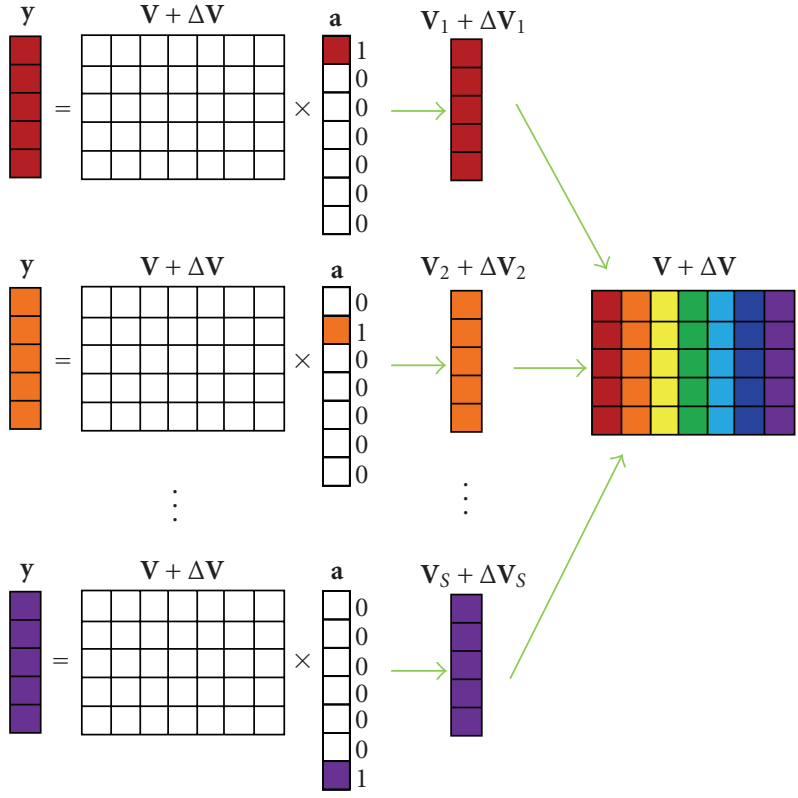

FIGURE 16: Illustration of the direct training approach to deal with the circuit imperfections.

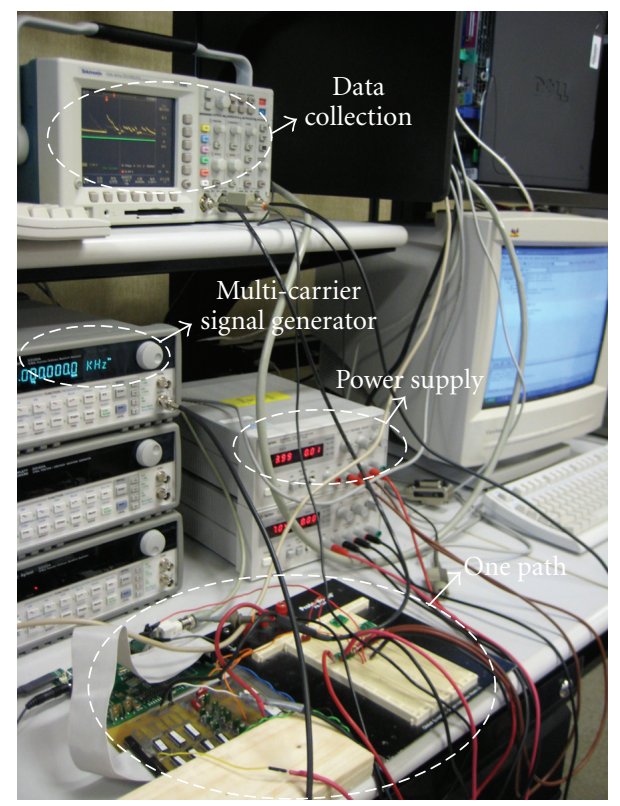

FIgURE 17: Testing setup of the prototype.

between the collected samples $\mathbf{y}$ and the coefficients a becomes

$$
\mathbf{y}=\tilde{\mathbf{V}} \mathbf{a}=(\mathbf{V}+\delta \mathbf{V}) \mathbf{a}
$$

where the element at the $m N+n$ row and the $s$ column of $\tilde{\mathbf{V}}$ is given by

$$
\widetilde{V}_{m N+n, s}=\int_{m T_{m}+\delta t_{1}}^{m T_{m}+T_{c}+\delta t_{2}} \alpha e^{j 2 \pi(s \Delta f+\delta f) t+\theta}\left(\Phi_{n}(t)+\delta \Phi_{n}(t)\right) d t .
$$

TABLE 1: Testing results of the prototype.

\begin{tabular}{lll}
\hline $\begin{array}{l}\text { Subcarrier's } \\
\text { amplitude } \\
(\mathrm{mV})\end{array}$ & $\begin{array}{l}\text { Subcarrier frequencies of } \\
\text { the input testing signal } \\
(\mathrm{kHz})\end{array}$ & $\begin{array}{l}\text { Subcarrier frequencies of } \\
\text { the reconstructed signal } \\
(\mathrm{kHz})\end{array}$ \\
\hline 0.3 & {$[+61,+121]$} & {$[+61,+121]$} \\
0.3 & {$[+41,+131]$} & {$[+41,+131]$} \\
0.3 & {$[+61,-131]$} & {$[+61,-131]$} \\
0.3 & {$[-51,+63,+111]$} & {$[-51,+63,+111]$} \\
0.2 & {$[+71,-85,+91,-101]$} & {$[+71,-85,+91,-101]$} \\
\hline
\end{tabular}

Here, $\delta t_{1}$ and $\delta t_{2}$ reflects the timing error on the slicing window, $\delta_{f}$ reflects the frequency offset, $\alpha$ and $\theta$ reflects the gain and phase mismatches, and the $\delta \Phi_{n}(t)$ reflects the error of the random basis which could be attributed to the jitter and nonzero response time.

Because the actual relationship between $\mathbf{y}$ and $\mathbf{a}$ is given by (15), we need to replace $\mathbf{V}$ with $\tilde{\mathbf{V}}$ in (1) when estimating a) otherwise, some extra error will be introduced. In [15], the authors discussed the impact of some circuit imperfections, such as the finite settling time of the PN sequences, and the timing uncertainty, and a background calibration algorithm based on LMS was proposed to compensate for the error due to these circuit nonideal factors. Because of the complexity of the background calibration, here we use a more simple approach based on direct training to deal with the circuit nonidealities. The direct training approach is illustrated in Figure 16. During the training stage, we inject a single-tone signal one at a time to the prototype and collect the samples from the 4 parallel paths, so that these samples will fill one column of the reconstruction matrix $\tilde{\mathbf{V}}$. After sending 100 single-tone signals, we obtain a complete matrix which will be used for signal reconstruction.

This pilot-based method is based on the assumption that the system is linear and time-invariant. Fortunately, our circuit level design ensures that the input signal swing is within the linear range of the system, and the microcontroller ensures that the system has the same initial condition for every run. Therefore, the linear time-variant assumption is reasonable. Implementing the background calibration for circuit imperfection compensation is part of our future work.

6.6. Testing Results. The testing setup for the prototype is shown in Figure 17. A series of experiments are done to test the functionality of the system. Table 1 summarizes the testing results, where + and - stand for the polarity of the BPSK modulation. Note that we scale the amplitude of each subcarrier according to the number of tones such that the amplitude of the multi-carrier signal is within the dynamic range of the system. From the testing results, the prototype achieves the design specification.

\section{Conclusions}

The Parallel Segmented Compressive Sensing (PSCS) frontend is able to sample and reconstruct analog sparse and compressive signals at sub-Nqyuist rate. The overlapping 
windowed integration in the PSCS front-end provides a spurious frequency rejection scheme by setting the lowpass filter nulls on the spurious frequencies without sacrificing the sampling rate requirement. A low-speed prototype is built with off-the-shelf components, which is able to sense sparse analog signals at sub-Nyquist rate.

\section{Acknowledgments}

The authors acknowledge the contributions of the students, faculty, and sponsors of the Analog and Mixed-Signal Center at the Texas A\&M University. This research project was funded under the DARPA Analog-to-Information Receiver Development Program (Army Research Laboratory Cooperative Agreement no. W911NF-08-2-0047) and the Army Research Laboratory Cooperative Technology Alliance (ARLCTA) contract no. DAAD-19-01-2-0011. Some of the results in this paper were reported in the conference of IEEE ICASSP'08, Las Vegas, Neveda, USA [11] and IEEE DCAS'09, Dallas, Texas, USA [15].

\section{References}

[1] J. Mitola III, Cognitive radio: an integrated agent architecture for software defined radio, Ph.D. dissertation, Royal Institute of Technology (KTH), May 2000.

[2] S. Haykin, "Cognitive radio: brain-empowered wireless communications," IEEE Journal on Selected Areas in Communications, vol. 23, no. 2, pp. 201-220, 2005.

[3] I. F. Akyildiz, W.-Y. Lee, M. C. Vuran, and S. Mohanty, "NeXt generation/dynamic spectrum access/cognitive radio wireless networks: a survey," Computer Networks, vol. 50, no. 13, pp. 2127-2159, 2006.

[4] Q. Zhao and B. M. Sadler, "A survey of dynamic spectrum access," IEEE Signal Processing Magazine, vol. 24, no. 3, pp. 7989, 2007.

[5] D. B. Cabric, Cognitive radios: system design perspective, Ph.D. dissertation, University of California, Berkeley, Calif, USA, December 2007.

[6] E. A. M. Klumperink, R. Shrestha, E. Mensink, V. J. Arkesteijn, and B. Nauta, "Polyphase multipath radio circuits for dynamic spectrum access," IEEE Communications Magazine, vol. 45, no. 5, pp. 104-112, 2007.

[7] R. H. Walden, "Performance trends for analog-to-digital converters," IEEE Communications Magazine, vol. 37, no. 2, pp. 96-101, 1999.

[8] D. L. Donoho, "Compressed sensing," IEEE Transactions on Information Theory, vol. 52, no. 4, pp. 1289-1306, 2006.

[9] E. J. Candès, J. Romberg, and T. Tao, "Robust uncertainty principles: exact signal reconstruction from highly incomplete frequency information," IEEE Transactions on Information Theory, vol. 52, no. 2, pp. 489-509, 2006.

[10] Z. Tian and G. B. Giannakis, "Compressed sensing for wideband cognitive radios," in Proceedings of the IEEE International Conference on Acoustics, Speech, and Signal Processing (ICASSP '07), vol. 4, pp. 1357-1360, Honolulu, Hawaii, USA, April 2007.

[11] Z. Yu, S. Hoyos, and B. M. Sadler, "Mixed-signal parallel compressed sensing and reception for cognitive radio," in Proceedings of the IEEE International Conference on Acoustics, Speech, and Signal Processing (ICASSP '08), pp. 3861-3864, Las Vegas, Nev, USA, March 2008.
[12] FCC, "Spectrum policy task force report," Tech. Rep. 02-135, ET Docket, 2002.

[13] M. Mishali and Y. C. Eldar, "Blind multi-band signal reconstruction: compressed sensing for analog signals," IEEE Transactions on Signal Processing, vol. 57, no. 3, pp. 993-1009, 2009.

[14] Y. C. Eldar, "Compressed sensing of analog signals in shiftinvariant spaces," IEEE Transactions on Signal Processing, vol. 57, no. 8, pp. 2986-2997, 2009.

[15] Z. Yu and S. Hoyos, "Digitally assisted analog compressive sensing," in Proceedings of the IEEE Dallas Circuits and Systems Workshop, 2009. 

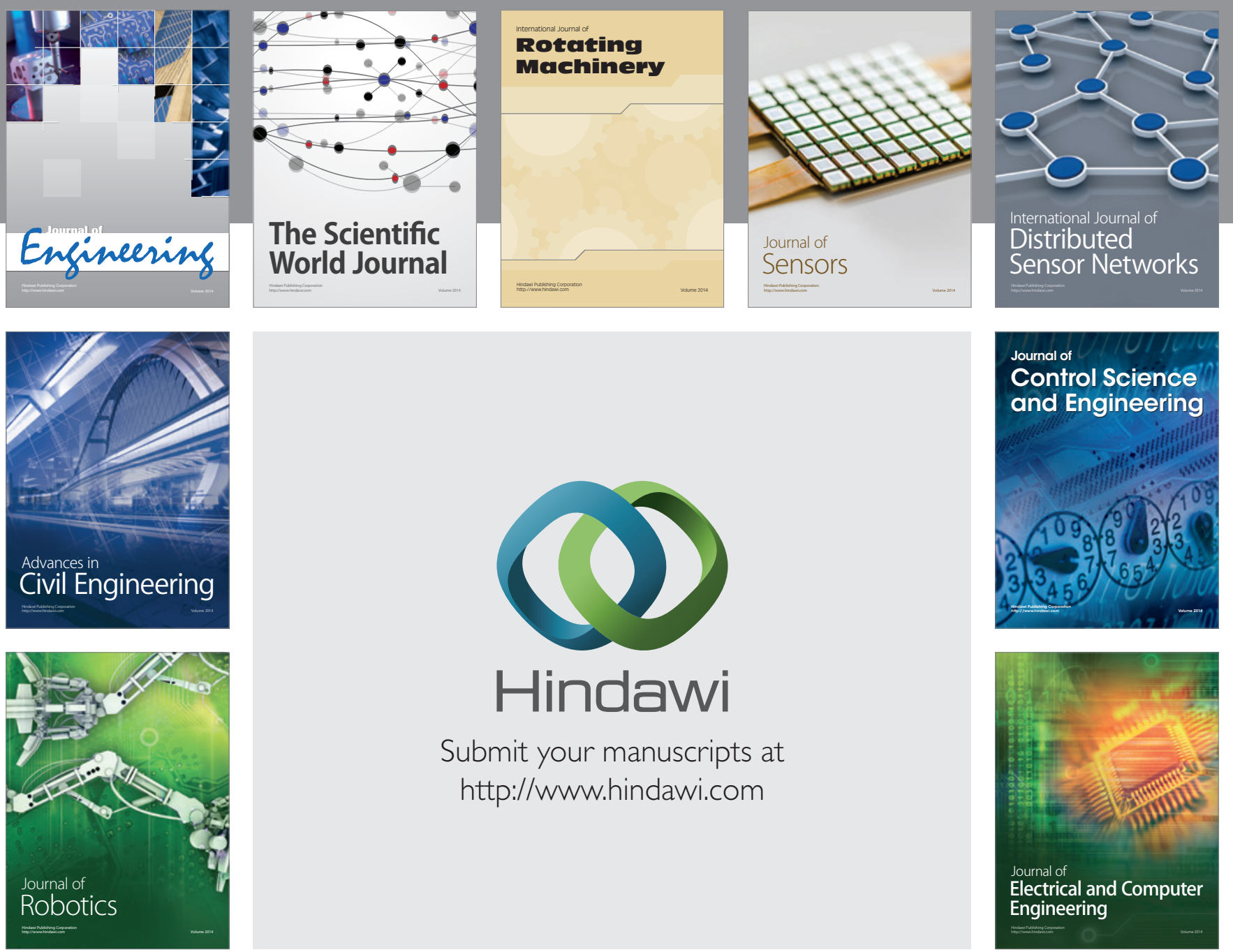

Submit your manuscripts at

http://www.hindawi.com
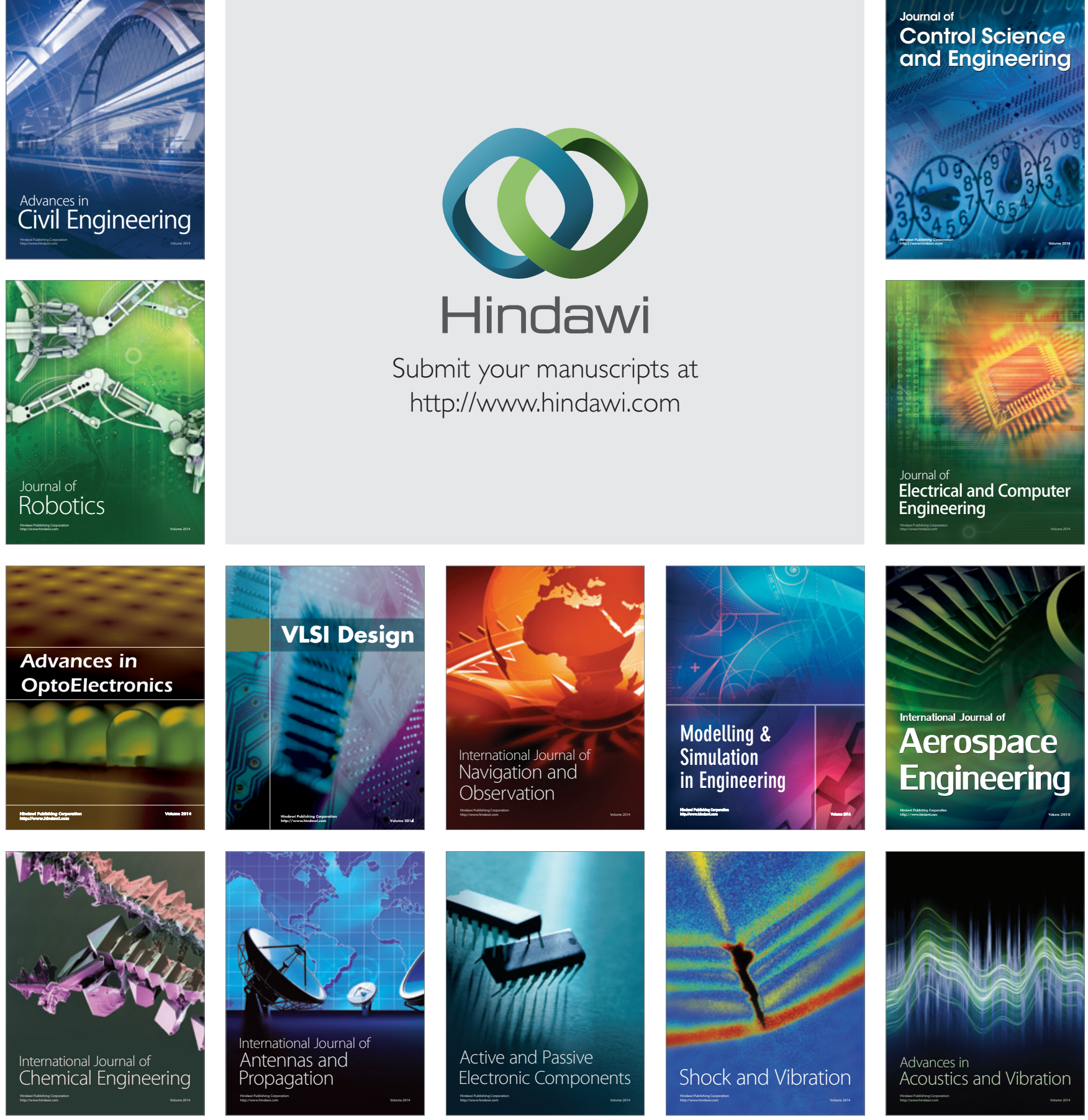